\title{
AVALIAÇÃO DA RELAÇÃO TERAPÊUTICA NA TERAPIA ASSÍNCRONA VIA INTERNET
}

\author{
Oliver Zancul Prado* \\ Sonia Beatriz Meyer"
}

\begin{abstract}
RESUMO. A terapia via Internet ainda não foi aprovada pelo Conselho Federal de Psicologia, portanto, pesquisas são recomendáveis. A possibilidade técnica de oferecer tal serviço e a formação da relação terapêutica foram investigadas. Foi desenvolvido um sistema de inscrição e psicoterapia breve, realizada de forma assíncrona. A relação terapêutica foi analisada por meio do Working Alliance Inventory (WAI). Os clientes que se inscreveram (373) eram predominantemente do sexo feminino, com a idade mediana de 28 anos, de nível superior e experientes no uso da Internet. Nas 53 terapias criadas, foram encontradas diferenças significativas, referentes ao WAI, entre os clientes que abandonaram a terapia (19) e os que a finalizaram (29). Foi possível realizar o serviço de terapia via Internet e a relação terapêutica desenvolvida apresentou características semelhantes às descritas na literatura a respeito do inventário.
\end{abstract}

Palavras-chave: psicoterapia, internet, relação terapêutica.

\section{EVALUATION OF THERAPEUTIC RELATIONS IN ASYNCHRONOUS THERAPY VIA INTERNET THROUGH WORKING ALLIANCE INVENTORY}

\begin{abstract}
The Brazilian Federal Council of Psychology has not approved Internet Therapy yet, therefore, research is recommended. The technical possibility of offering such a service and the possibility of developing a working alliance were investigated. A system of registration and brief psychotherapy, which was asynchronously carried out, was created using free software. The therapeutic relationship was analyzed by using the Working Alliance Inventory (WAI). Clients enrolled (373) were predominantly female, with median age around 28, graduate and experienced Internet users. In the 53 therapies carried out, there were significant differences in the WAI results between clients who abandoned the therapy (19) and clients who finished it (29). It was possible to conduct Internet therapy, and the therapeutic relationship presented characteristics similar to those described in the specialized bibliography about the inventory.
\end{abstract}

Key words: Psychotherapy, internet, Working Alliance.

\section{EVALUACIÓN DE LA RELACIÓN TERAPÉUTICA EN LA TERAPIA ASÍNCRONA VÍA INTERNET}

\begin{abstract}
RESUMEN. Como la terapia vía Internet aún no ha sido aprobada por el Consejo Federal de Psicología, investigaciones son recomendables. La posibilidad técnica de ofrecer tal servicio y la formación de la relación terapéutica fueron investigadas. Se desarrolló un sistema de inscripción y psicoterapia breve, realizada asincrónicamente. La relación terapéutica fue analizada a través del Working Alliance Inventory (WAI). Los pacientes inscritos (373) eran predominantemente mujeres, con edad media de 28 años, graduadas y usuarias de Internet. En las 53 terapias creadas, había diferencias significantes en los resultados de WAI entre pacientes que abandonaron la terapia (19) y los que la terminaron (29). Fue posible realizar el servicio de terapia vía Internet, y la relación terapéutica desarrollada presentó características similares a las descritas en la literatura sobre el inventario.
\end{abstract}

Palabras-clave: psicoterapia, internet, relación terapéutica.

\footnotetext{
* Mestre em Psicologia Clínica e Professor da Universidade Paulista.

\# Doutora em Psicologia Experimental e Professora da Universidade de São Paulo.
} 
A psicoterapia, segundo o Conselho Federal de Psicologia-CFP (2000), é uma das diversas práticas do psicólogo, constituindo-se em um processo científico de compreensão, análise e intervenção realizada por meio da aplicação de métodos e técnicas psicológicas que visa propiciar ao cliente condições para que ele possa enfrentar seus conflitos e/ou transtornos psíquicos.

A Psicologia, por sua vez, possui diversas escolas, como, por exemplo, a Behaviorista, a de Psicanálise e a da Gestalt (Keller, 1983), as quais possuem modelos científicos, filosóficos, teóricos e metodológicos distintos. No entanto, a definição de psicoterapia utilizada pelo CFP tenta isentar-se das visões específicas de cada escola da psicologia, na medida em que define o serviço prestado pelo psicólogo como profissional e sujeito à legislação vigente no Brasil e às resoluções do CFP.

As escolas de psicologia têm grandes diferenças entre si, porém a relação terapêutica está presente em todas as abordagens de psicoterapia existentes, apesar de ser analisada e entendida de maneiras distintas.

Ao pensar em atendimento realizado pela Internet, não há dúvidas de que um dos principais desafios é saber se isso é psicoterapia e, mesmo antes disso, se ocorre uma de suas condições básicas, ou seja, se se formará uma relação terapêutica sem que haja qualquer tipo de contato presencial face a face. Se verificarmos que a relação se forma via Internet, será possível considerar a realização de procedimentos terapêuticos à distância. Para a avaliação da relação terapêutica deveriam ser usados os mesmos instrumentos de medida que a avaliaram e consagraram na psicoterapia presencial.

\section{A AVALIAÇÃo DA ALIANÇA TERAPÊUTICA}

Buscando na literatura maneiras de avaliar a relação terapêutica, Meyer e Vermes (2001) encontraram dois conjuntos de instrumentos:

1. Questionários respondidos pelos clientes e/ou terapeutas.

2. Observação e análise das sessões com enfoque em aspectos da relação terapêutica com sistemas de categorização.

Desses dois conjuntos de instrumentos, o primeiro possui uma metodologia mais definida de investigação e já registra resultados concretos e consagrados na literatura sobre psicoterapia.

Embasando essa afirmação, um estudo de revisão bibliográfica realizado por Luborsky (1994) concluiu que questionários que medem a aliança terapêutica são significativamente preditivos de resultados em terapia. Horvath e Symonds (1991) fizeram uma metanálise, encontrando uma associação moderada, mas confiável, entre boa aliança terapêutica e bons resultados. Os autores concluíram que, no geral, a qualidade da aliança terapêutica foi mais preditiva de resultados de tratamento quando baseada na avaliação dos clientes, seguida da avaliação dos terapeutas e, por último, de avaliações de observadores.

Se há evidências empíricas suficientes relatando que existe relação entre aliança terapêutica e resultados, em que momento da terapia a relação deveria ser avaliada? Dados da literatura mostram que a avaliação feita a partir do uso de questionários na terceira ou quarta sessão parece indicar, de forma suficiente, predições de resultados (Luborsky, 1994; Garfield, 1995). Horvath (1994) também argumentou a favor da avaliação da aliança nos estágios iniciais da terapia como melhor forma de predição de resultados, além de ressaltar que a avaliação no início da terapia também permite ao terapeuta ou pesquisador saber quais clientes poderão abandoná-la futuramente.

\section{WORKING ALLIANCE INVENTORY (WAI)}

O WAI (Horvath \& Greenberg, 1989) é considerado por seu idealizador como um instrumento metateórico, ou seja, que pode ser utilizado para avaliar a aliança terapêutica independentemente da abordagem teórica, que consiste num questionário (com versões para cliente, terapeuta e observador) que apresenta três subescalas com doze questões cada, sendo que as questões são avaliadas num espectro de sete itens (sempre - nunca).

As subescalas são: Objetivos, subescala que se caracteriza pela negociação e entendimento mútuo entre terapeuta e cliente acerca dos objetivos da terapia em termos de resultados; Tarefa, que se refere a atividades específicas desenvolvidas pelo terapeuta e cliente para instigar ou facilitar as mudanças; e Vínculo, que se refere a ligações interpessoais entre cliente e terapeuta que se desenvolvem na atividade compartilhada da terapia. É expresso e sentido em termos de amizade, simpatia, confiança, respeito pelo outro e um senso de comprometimento comum e um entendimento compartilhado das atividades.

Essas escalas são altamente correlacionadas (Horvath \& Greenberg, 1989; Horvath, 1994), indicando que elas abordam aspectos da aliança que estão fortemente interligados.

O WAI foi o instrumento escolhido, e as razões para sua escolha foram: a) a versão autorizada em português produzida por Paulo Machado e Cristiano 
Nabuco de Abreu; b) esse instrumento estar entre os que mais apareceram na literatura encontrada sobre instrumentos de avaliação da relação terapêutica, c) a possibilidade de utilizar o instrumento numa terapia em que não ocorrem sessões propriamente ditas, já que ele não aborda as sessões e, sim, os objetivos, tarefas e vínculo entre terapeuta e cliente, considerando que a maioria dos outros instrumentos encontrados avaliavam ou abordavam as sessões de terapia; d) o amplo respaldo na literatura sobre a validade do instrumento (Horvath,1994) relata que um grande número de estudos realizados separadamente provêm suporte à validade do WAI); e) o fato de que a confiabilidade do instrumento, baseada na homogeneidade (Alpha de Cronbach) entre os itens vai de .84 a .93 e entre as subescalas, de .68 a .92 (Horvath, 1994).

\section{Alguns estudos sobre terapia via Internet}

Existem já algumas pesquisas preliminares relativas à Terapia On Line, termo adotado por King (1998) para definir o contato à distância com objetivos terapêuticos. Sander (1996) propôs a um grupo de clientes em fase terminal de terapia e familiarizados com computadores que realizassem três sessões via computador. Sugeriu que a comunicação assíncrona pode ser mais vantajosa, na medida em que evita a fragmentação do discurso e permite que cada membro se conecte ao sistema e envie mensagens em horários considerados convenientes.

Barak e Wander-Schwartz (1999) conduziram uma pesquisa cujos objetivos eram os de comparar a utilidade de um grupo de terapia via chat com um grupo com terapia-padrão e um grupo-controle não tratado avaliando: impacto da terapia na autoestima, relações sociais e bem-estar, processos grupais, e utilizando um questionário de avaliação específico para o estudo. Relatou que não foram encontradas diferenças significativas entre o grupo de chat e o grupo de terapia-padrão, concluindo com cautela que o chat em grupo pode ser usado com finalidades terapêuticas.

Day e Schneider (2002) realizaram um estudo comparando terapia face a face, por videoconferência e por voz, encontrando pequenas diferenças relativas ao processo e resultados entre os grupos pesquisados.

É importante ressaltar o trabalho de Cook (2001), por ser semelhante a essa pesquisa. Ele realizou um estudo sobre relação terapêutica e terapia via Internet nas diferentes formas de comunicação. Nesse estudo, terapeutas que realizavam terapia via Internet (como um serviço profissional) forneciam aos seus clientes um login e uma senha para que estes respondessem ao WAI, concluindo que a relação terapêutica pode ser fortemente estabelecida entre aqueles que consideram a terapia via Internet um método atraente para receber assistência em saúde mental. Este estudo possuía limitações sobre o controle amostral, pois os terapeutas escolhiam os clientes que iam participar do estudo, e também não houve controle sobre o tempo em que esses clientes estavam fazendo terapia. Todavia, esses resultados são um primeiro indicativo de que a relação terapêutica pode ser formada e mantida via Internet.

\section{MÉTODO}

\section{Participantes}

\section{Terapeutas}

Participaram como terapeutas psicólogos com pelo menos quatro anos de experiência clínica (incluindo os estágios da graduação) e com conhecimentos básicos de uso da Internet, bem como de ferramentas de comunicação mediada pelo computador, que aceitaram os termos de contrato para realizar a terapia durante quinze semanas e que possuíam computador e sistemas compatíveis com as ferramentas utilizadas no estudo.

\section{Clientes}

Como clientes participaram indivíduos maiores de idade, com pleno acesso à Internet e com experiência no uso da mesma, que responderam ao questionário de anamnese e aos formulários enviados via correio e aceitaram e assinaram os termos de contrato terapêutico e consentimento e que possuíam computador e sistemas compatíveis com as ferramentas utilizadas no estudo.

\section{Critérios de exclusão}

Constituíram critérios de exclusão o cliente ter mencionado na anamnese tendências suicidas ou tentativas de suicídio prévias ao tratamento, ter citado na anamnese o uso diário de drogas psicotrópicas ou ingestão superior a duas doses de álcool/dia, estar sob algum outro tipo de tratamento psicológico ou psiquiátrico, ter sido excedido o número máximo de participantes do estudo (nos dois primeiros casos seria feito encaminhamento a um profissional ou instituição da localidade do participante, caso solicitado). 


\section{Material}

\section{Softwares e Hardwares}

Para realizar a terapia via Internet, foi criado um website contendo informações básicas sobre a pesquisa, pesquisadores e instituição (USP), além de uma cópia do consentimento informado (que também era enviada pelo correio para os que se inscreveram na pesquisa), da Resolução CFP No 003/2000 sobre atendimento mediado pelo computador, da Resolução do CNS 196/96 sobre pesquisas com seres humanos e do documento de aprovação da pesquisa pelo Comitê de Ética da Faculdade de Saúde Pública da USP (cópia em formato .gif ).

Para a realização das terapias foi utilizada uma versão modificada do software Learnloop ${ }^{1}$ originalmente criado para educação à distância. Também foi utilizada uma versão informatizada do WAI integrada ao Learnloop modificado. O computador servidor rodava o sistema operacional Linux e os computadores clientes deveriam ter conexão à Internet e um navegador da geração 4.x ou superior instalado para acessar o web site e o Learnloop. Todos os dados enviados pelos participantes ficavam armazenados em uma base de dados no computador servidor.

\section{Divulgação e inscrição}

Após a criação do website da pesquisa e a obtenção do aval do comitê de ética em pesquisa, a divulgação foi iniciada. $O$ procedimento da divulgação foi diferente para terapeutas e clientes. Para terapeutas a divulgação foi feita de forma específica e direcionada, enquanto para os clientes foi feita de forma não direcionada, possibilitando abranger internautas em geral. Para os terapeutas a pesquisa foi divulgada através do envio de $e$-mails para listas de discussão sobre psicologia e para psicólogos conhecidos do pesquisador; para os clientes a pesquisa foi divulgada através de uma notícia publicada do boletim da Agência de Notícias da USP, notícia que por sua vez foi replicada por veículos de mídia.

A inscrição foi feita por intermédio do web site da pesquisa, o qual disponibilizava o texto do termo de consentimento antes de o participante enviar qualquer informação. A inscrição constituía-se de duas partes; a primeira continha informações demográficas, de identificação e de uso de Internet,

\footnotetext{
Http://www.learnloop.org
}

e a segunda, a opção por inscrição para clientes ou inscrição para terapeutas. $\mathrm{Na}$ inscrição para clientes era preenchido um questionário de história de vida (anamnese) e na inscrição para terapeutas, um questionário de experiência profissional.

Após preencher todos os formulários, os participantes recebiam um e-mail contendo as informações para entrar no sistema, e após a realização do login no sistema eram enviadas correspondências pelo correio para os participantes, que tinham que assinar o termo de consentimento e anexar uma fotocópia do RG (clientes) ou CRP (terapeutas) e então retornar a correspondência para o endereço do pesquisador.

\section{O processo de terapia}

Após todos os procedimentos de inscrição serem concluídos, tanto os clientes quanto os terapeutas recebiam uma mensagem por e-mail contendo instruções para a terapia.

A terapia ocorria de forma assíncrona, ou seja, num fórum de discussão. As mensagens, portanto, eram enviadas pelos participantes em horários diferentes, minimizando possíveis problemas de conexão à Internet. Sendo assim, não existiu a delimitação da sessão, como ocorre na terapia presencial ou síncrona. Além disso, não havia dia nem horário determinados para postar mensagens. Basicamente, o usuário entra no sistema, lê as mensagens e envia a sua.

As regras da terapia descreviam que essa deveria durar 15 semanas, duração comum de terapias breves, sendo contabilizadas apenas as semanas em que o cliente entrava no sistema. Dessa forma, quando o cliente ficava mais de uma semana sem entrar no sistema, uma falta era contabilizada, porém não era contabilizada uma semana de terapia. A partir dessa forma de contar as semanas, foi possível garantir um total de 15 semanas de participação na terapia, mesmo que o total de semanas consecutivas fosse superior a 15 .

Uma mensagem para terapeuta e clientes responderem ao WAI era postada no fórum na $5^{\mathrm{a}}$, $10^{\mathrm{a}}$ e $15^{\mathrm{a}}$ semana de terapia ${ }^{2}$ (com intervalos de até duas semanas de diferença). $\mathrm{Na} 15^{\mathrm{a}}$ semana também era postada uma mensagem para responder ao questionário final (que não será analisado nesse artigo), juntamente com o aviso de que a terapia estava terminada, mas que seria aguardado o fechamento e haveria a possibilidade

2 Respectivamente denominado: $1^{\circ}, 2^{\circ}$ e $3^{\circ}$ momento 
de mantê-la por mais algum tempo (uma ou duas semanas).

Ao final da terapia, a sala era desativada. Se a terapia terminasse antes da $15^{\mathrm{a}}$ semana por abandono ou por desejo do cliente, a sala também era desativada. Abandonos aconteciam quando um dos participantes ficava quatro semanas sem entrar no sistema, sem responder aos contatos via $e$-mail do terapeuta ou pesquisador ou então relatava o desejo de interromper a terapia.

\section{RESULTADOS}

\section{Inscrições e Participação}

Ao todo, 373 pessoas inscreveram-se para participar da terapia via Internet como clientes e 20 como terapeutas, sendo estes de diversas abordagens teóricas. Porém, da mesma forma que em outras pesquisas e atendimentos clínicos, houve vários níveis de desistência, tendo 161 clientes e 15 terapeutas realizado login no sistema e 86 clientes e 16 terapeutas retornado a correspondência pelo correio (um dos 20 terapeutas retornou a correspondência sem ter feito o login).

Por conta de restrições temporais das 86 terapias possíveis, foram criadas 53 salas de terapia, das quais 29 foram concluídas, 23 não foram finalizadas e uma teve que ser finalizada por falta de tempo hábil para a pesquisa. Todas as salas que não finalizaram foram consideradas como casos de abandono, independentemente de quando isso tenha ocorrido e do motivo. Não foram feitas diferenciações entre as terapias em que o abandono ocorreu sem aviso ao terapeuta ou pesquisador e as que terminaram precocemente com o aviso do cliente.

\section{Dados demográficos}

O maior percentual de clientes era proveniente do Estado de São Paulo (32\%), ficando o Estado do Rio Grande do Sul em segundo lugar (19\%). Contudo, verificou-se uma grande diversidade na localidade geográfica dos clientes, os quais eram provenientes de quase todos os Estados brasileiros (exceto Acre, Roraima e Tocantins). Mesmo a divulgação tendo sido focada no Brasil, houve a ocorrência de alguns clientes provenientes de localidades internacionais.

A grande maioria dos clientes era do sexo feminino $(70 \%)$ e possuía alto grau de escolaridade, sendo que quase $80 \%$ possuíam pelo menos $3^{\circ}$ grau incompleto. Cerca de $55 \%$ eram solteiros e a idade mediana era de 28 anos. Cerca de $77 \%$ dos clientes tinham mais de dois anos de experiência no uso de Internet.

\section{Aliança Terapêutica}

Para aceitar ou rejeitar a hipótese da formação e manutenção da aliança terapêutica na terapia via Internet, foi preciso seguir determinados critérios. Um deles era o de analisar se os resultados do WAI eram similares àqueles encontrados na literatura sobre avaliação da relação terapêutica. Essa análise foi feita com base a) no momento em que se forma a relação; b) na manutenção da relação no decorrer do processo de terapia; e c) nas diferenças entre terapeutas e clientes relativamente à predição de resultados.

Outro critério era o de analisar se as propriedades psicométricas do WAI na amostra desta pesquisa eram similares às que foram descritas na literatura, já que a descoberta de resultados semelhantes seria mais um indicativo de que o instrumento comporta-se na terapia via Internet de forma similar à das terapias reconhecidas.

Após o término de todas as terapias, os dados foram agrupados em quatro grupos diferentes ${ }^{3}$ : a) clientes que terminaram a terapia (CT); b) terapeutas das salas que terminaram a terapia (TT); c) clientes que não terminaram a terapia, isto é, que abandonaram ou foram abandonados (CA); d) terapeutas das salas que não terminaram a terapia, isto é, que abandonaram ou foram abandonados (TA). Na Tabela 1 os números dos grupos (n) descrevem a dupla terapeuta/cliente, sendo que o $n$ dos clientes é igual ao número efetivo de pessoas participantes, mas o $n$ dos terapeutas, não, já que a grande maioria dos terapeutas atendeu mais do que um cliente. Não foram criados grupos diferentes com o critério de quem abandonou a terapia (terapeuta ou cliente), pois o número de quatro terapias, nas quais quem abandonou foram os terapeutas, não foi suficiente para ser considerado uma amostra para os testes estatísticos realizados.

Com relação às 23 terapias que não terminaram, 11 clientes responderam ao WAI da $5^{\mathrm{a}}$ semana de terapia.

A Tabela 1 mostra médias e medianas do WAI e das subescalas dos quatro grupos analisados. Nota-se que o grupo $\mathrm{CA}$ tem as menores medianas do WAI (4,08), comparativamente aos outros três grupos, em que o menor valor foi 5,50 e o maior, 5,92.

\footnotetext{
Para fazer os testes estatísticos, os participantes que não responderam ao WAI em algum momento da aplicação tiveram que ser excluídos da análise.
} 
Tabela 1. Médias, Medianas e Desvio Padrão para os Resultados do WAI e Subescalas

\begin{tabular}{lccccccccccccc}
\hline & \multicolumn{3}{c}{ CT $(\boldsymbol{n}=\mathbf{2 8})$} & \multicolumn{4}{c}{ CA $(\boldsymbol{n}=\mathbf{1 1})$} & \multicolumn{3}{c}{ TT $(\boldsymbol{n}=\mathbf{2 5})$} & \multicolumn{3}{c}{ TA $(\boldsymbol{n}=\mathbf{1 0})$} \\
\cline { 2 - 13 } & $\boldsymbol{M}$ & $\boldsymbol{M d n}$ & $\boldsymbol{S D}$ & $\boldsymbol{M}$ & $\boldsymbol{M d n}$ & $\boldsymbol{S D}$ & $\boldsymbol{M}$ & $\boldsymbol{M d n}$ & $\boldsymbol{S D}$ & $\boldsymbol{M}$ & $\boldsymbol{M d n}$ & $\boldsymbol{S D}$ \\
\hline $1^{\text {o }}$ Momento & & & & & & & & & & & & \\
Vínculo & 5,79 & 5,92 &, 85 & 4,63 & 4,58 &, 74 & 5,96 & 6,00 &, 56 & 6,08 & 6,17 &, 64 \\
Tarefa & 5,41 & 5,75 & 1,04 & 3,85 & 3,83 & 1,33 & 5,42 & 5,33 &, 74 & 5,23 & 5,21 & 1,04 \\
Objetivos & 5,05 & 5,33 &, 97 & 3,60 & 3,42 & 1,10 & 4,93 & 4,75 &, 92 & 4,90 & 4,80 & 1,00 \\
WAI & 5,40 & 5,60 & 1,00 & 4,03 & 4,08 & 1,14 & 5,44 & 5,50 &, 86 & 5,41 & 5,43 &, 83 \\
\hline
\end{tabular}

$2^{\circ}$ Momento

\begin{tabular}{lllllll} 
Vínculo & 5,88 & 5,96 &, 85 & 6,09 & 6,08 &, 64 \\
Tarefa & 5,61 & 5,88 &, 97 & 5,52 & 5,58 &, 91 \\
Objetivos & 5,31 & 5,50 &, 95 & 5,22 & 5,50 &, 99 \\
WAI & 5,60 & 5,70 &, 90 & 5,61 & 5,75 &, 92 \\
\hline
\end{tabular}

$3^{\circ}$ Momento

$\begin{array}{lcccccc}\text { Vínculo } & 5,81 & 5,83 & , 94 & 6,14 & 6,08 & , 64 \\ \text { Tarefa } & 5,54 & 5,67 & 1,10 & 5,71 & 5,67 & , 93 \\ \text { Objetivos } & 5,20 & 5,17 & , 98 & 5,35 & 5,25 & , 95 \\ \text { WAI } & 5,50 & 5,80 & 1,00 & 5,73 & 5,92 & , 90\end{array}$

Nota: $\quad \mathrm{CT}=$ Clientes que Terminaram, $\mathrm{TT}=$ Terapeutas que Terminaram $(n=14), \mathrm{CA}=$ Clientes Abandonaram, TA $=$ Terapeutas Abandonaram $(n=7) .1^{\circ}, 2^{\circ}$ e $3^{\circ}$ Momentos correspondem às $5^{\mathrm{a}}, 10^{\mathrm{a}}$ e $15^{\mathrm{a}}$ semanas de terapia respectivamente. Os $n$ da Tabela correspondem às salas de terapia.

Os dados indicam que os valores de CT e TT são similares, enquanto os valores de CA e TA, não, levantando a questão de que possivelmente os resultados do WAI dos clientes foram melhores preditores de participação na terapia do que os resultados dos terapeutas.

Observam-se, ainda, variações entre as subescalas em todos os grupos e, por outro lado, poucas variações entre cada subescala nos diferentes momentos.

Os dados dessa tabela são suficientes para indicar que a intensidade da relação terapêutica entre os participantes que terminaram a terapia foi alta, pois as medianas variaram entre 5,5 e 5,9, ou seja, com relação à classificação das respostas do WAI (de 1 a 7 - nunca a sempre), a avaliação da relação terapêutica variou entre freqüentemente e muito freqüentemente.

Para responder às outras questões outros testes estatísticos foram realizados. Primeiramente foram verificadas as correlações das subescalas entre todos os grupos, e para os grupos CT e TT calcularam-se também as correlações entre o WAI nos três momentos. O teste utilizado foi $\mathrm{o}$ de Pearson. Em todas as análises foram encontradas correlações variando entre moderadas e fortes. Não foi encontrado, nos diversos cruzamentos, nenhum valor $P$ igual ou maior do que 0.05 , o que comprova a hipótese de que há correlações estatisticamente significativas para todas as variáveis. Para os dois grupos de clientes, as correlações variam entre 0.462 e 0.889 e, para os dois grupos de terapeutas, variam entre 0.511 e 0.933 .

Para verificar se existia diferença significativa entre os quatro grupos, entre as subescalas e entre os momentos, foi escolhida a Análise de Variância (ANOVA), que é a técnica adequada para verificar se há diferença, em um tratamento realizado, entre as médias das populações.

Dessa forma, foram realizadas ANOVAS com os seguintes fatores:

- momentos e subescalas para CT e TT (cada grupo individualmente);

- grupos, subescalas e momentos, comparando os grupos CT x TT;

- grupos e subescalas, comparando os grupos CT x CA, TT x TA e CA x TA.

A Tabela 2 mostra os valores da estatística-teste (F) e os graus de liberdade (df) das ANOVAS, que 
foram realizadas com os quatro grupos analisados. Como não foi encontrado nenhum cruzamento (interação) entre os fatores analisados, apenas o cruzamento dos fatores listados acima foi demonstrado na tabela. Nota-se que existiram diferenças signifi- cativas entre as escalas $(\mathrm{P}<0.05)$ em todos os cruzamentos entre grupos e intragrupos. Todavia, com este teste não foi possível discriminar qual subescala é diferente das outras, e sim, que pelo menos uma delas é diferente.

Tabela 2. Análise de Variância para os Quatro Grupos de Participantes

\begin{tabular}{|c|c|c|c|c|c|c|c|c|}
\hline & \multicolumn{4}{|c|}{ Terminaram } & \multicolumn{4}{|c|}{ Abandonaram } \\
\hline & \multicolumn{2}{|c|}{$\begin{array}{c}\text { Clientes } \\
(n=28)\end{array}$} & \multicolumn{2}{|c|}{$\begin{array}{c}\text { Terapeutas } \\
(n=25)\end{array}$} & \multicolumn{2}{|c|}{$\begin{array}{l}\text { Clientes } \\
(n=11)\end{array}$} & \multicolumn{2}{|c|}{$\begin{array}{c}\text { Terapeutas } \\
(n=10)\end{array}$} \\
\hline & $\mathrm{df}$ & $F$ & $\mathrm{df}$ & $\mathrm{F}$ & df & $\mathrm{F}$ & $\mathrm{df}$ & $\mathrm{F}$ \\
\hline \multicolumn{9}{|l|}{$\mathrm{CT}$} \\
\hline Momentos & 2 &, 78 & 2 & 1,69 & - & - & & \\
\hline Subescalas & 2 & $10.15^{* * *}$ & 2 & $20.90 * * *$ & 2 & $6.21 * *$ & & \\
\hline Grupo & - & - & 1 &, 61 & 1 & $60.96 * * *$ & & \\
\hline \multicolumn{9}{|l|}{ TT } \\
\hline Momentos & & & 2 & 2,66 & - & - & - & - \\
\hline Subescalas & & & 2 & $21,66 * * *$ & 2 & $6.41 * *$ & 2 & $1.40 * * *$ \\
\hline Grupo & & & - & - & 1 & $25.86 * * *$ & 1 & ,03 \\
\hline
\end{tabular}

Nota. $\mathrm{CT}=$ Clientes que Terminaram, $\mathrm{TT}=$ Terapeutas que Terminaram

Foram omitidos os cruzamentos não significativos que verificavam as interações entre os fatores.

No cruzamento entre CT e TT, CT tem $(n=25)$. O números dos terapeutas são relativos às salas atendidas.

** $\mathrm{P}<0.05$

$* * * \mathrm{P}<0.01$

Em relação aos três diferentes momentos de aplicação do WAI, nas análises intragrupo não foram verificadas diferenças entre os grupos CT e TT e nas análises entre esses grupos também não foram encontradas diferenças. Já comparando-se os grupos CA, CT, TA e TT, verificou-se diferença entre o grupo $\mathrm{CA}$ e os demais

De forma resumida, os resultados mostraram que para todos os grupos há diferenças entre as subescalas, que para os que terminaram a terapia (clientes e terapeutas) não há diferenças nos três momentos de aplicação do WAI e que na comparação entre os quatro grupos, apenas o grupo CA foi diferente dos demais.

A diferença entre as subescalas verificada em todas as análises poderia levantar dúvidas quanto à consistência do WAI, fato que já foi refutado na validação do instrumento com terapias tradicionais, mas não com terapias via Internet.

Para responder a essa pergunta, foi utilizada a técnica de Cronbach, que é um teste que leva em consideração o número de itens das subescalas, a covariância, a variância e a correlação média entre os itens. Tal teste pode ser interpretado como um coeficiente de correlação. Como a correlação entre as subescalas (já calculadas) foi alta, era esperado um alpha alto, ou seja, como o alpha varia entre 0 e 1 , era esperado que esse fosse próximo de 1.

Para a realização desse teste, foi escolhido o grupo CT, pois era o que apresentava o maior número de sujeitos participantes e o que obteve os melhores resultados preditivos de participação na terapia. Foram utilizadas as três subescalas nos três momentos, tendo sido obtido um alpha de.9287 para o primeiro momento e .8930 e .9203 para os outros momentos respectivamente. Esses resultados indicam que a consistência interna do WAI também foi alta quando verificada na terapia via Internet.

Os gráficos da Figura 1 fornecem as seguintes informações: a) mediana, que são os traços dentro de cada caixa apresentada no gráfico; b) variabilidade, que são os tamanhos das caixas que contêm $50 \%$ da amostra; c) pontos de máximo, que são os traços acima das caixas; d) pontos de mínimo, que são os traços abaixo das caixas. Os asteriscos representam outliers (resultados discrepantes). 

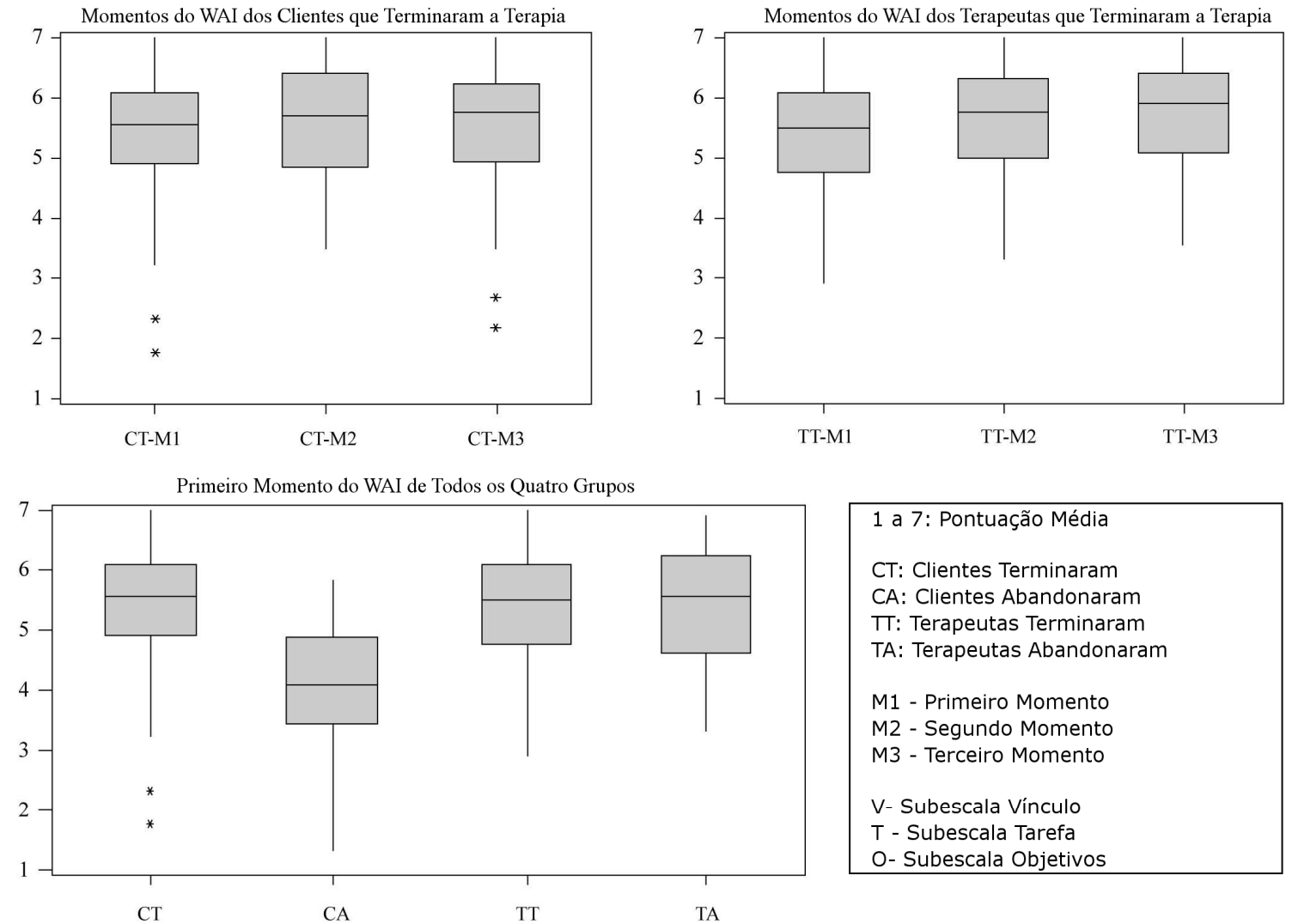

\begin{tabular}{|l|}
\hline 1 a 7: Pontuação Média \\
CT: Clientes Terminaram \\
CA: Clientes Abandonaram \\
TT: Terapeutas Terminaram \\
TA: Terapeutas Abandonaram \\
M1 - Primeiro Momento \\
M2 - Segundo Momento \\
M3 - Terceiro Momento \\
V- Subescala Vínculo \\
T - Subescala Tarefa \\
O- Subescala Objetivos
\end{tabular}
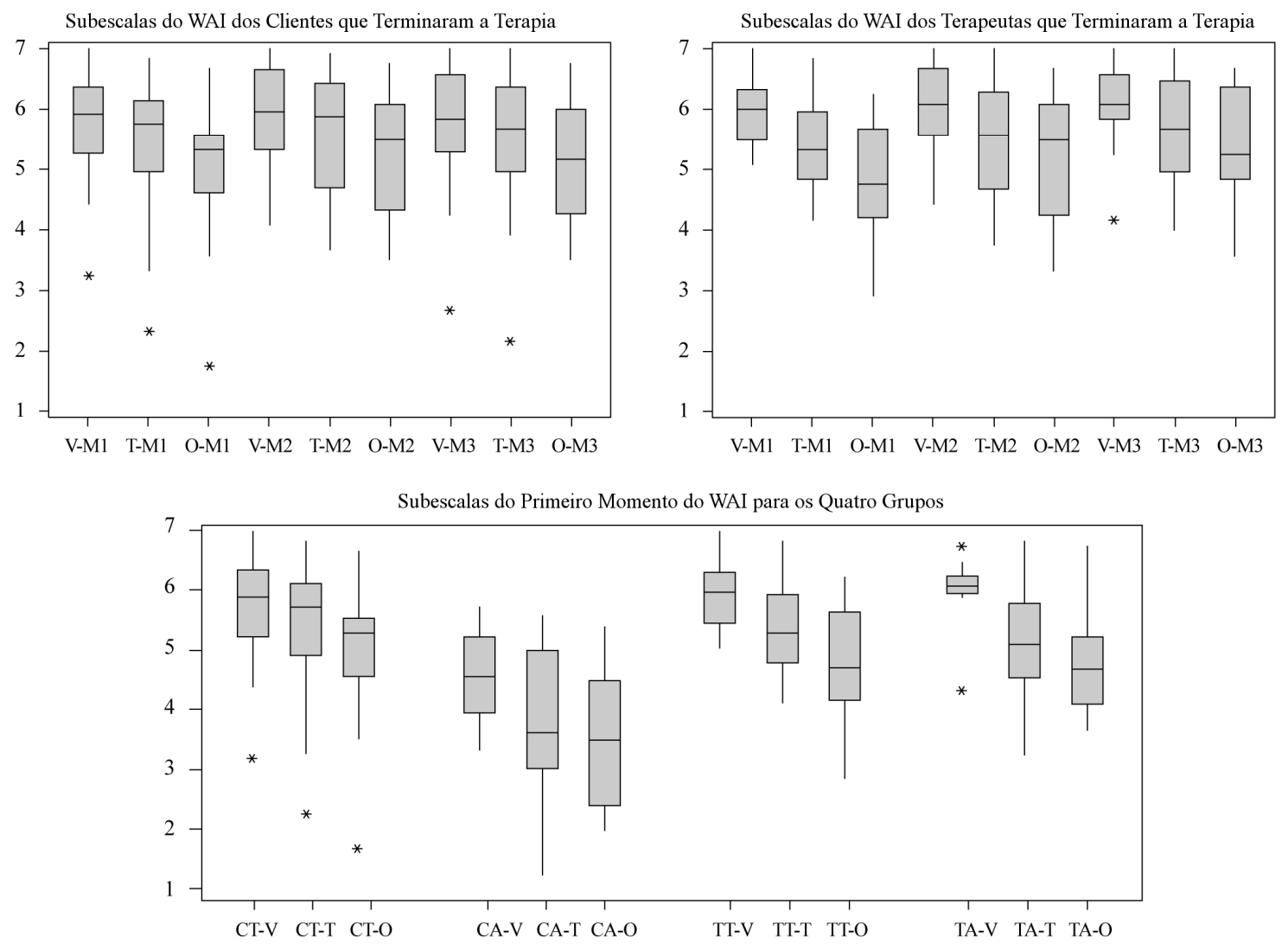

Figura 1. Box Plot dos Resultados do WAI e das Subescalas dos Quatro Grupos 
Ao observar os gráficos dos grupos CT e TT é possível visualizar com clareza o que foi analisado nos testes estatísticos. Observa-se um padrão semelhante entre o WAI nos três momentos e também entre as subescalas ao longo dos três momentos, tanto para clientes como para terapeutas, sendo que em ambos os grupos a escala-vínculo tem valores mais altos nos três momentos.

Os gráficos de comparação entre os quatro grupos evidencia claramente a diferença nos resultados do WAI entre o grupo CA e os demais, apesar de todos os grupos manterem o mesmo padrão em relação às subescalas, ou seja, a subescala Vínculo tem valores mais altos, seguida pela subescala Tarefa e, finalmente, pela Objetivos. Os gráficos mostram que a avaliação dos grupos CA e TA foi diferente. Ao contrário dos clientes, que apresentaram diferenças entre os grupos dos que terminaram a terapia e os que a abandonaram, os terapeutas avaliaram de forma similar as terapias das salas que terminaram e das salas que abandonaram o processo contratado.

Ao observar os valores da subescala Vínculo no grupo TA, nota-se uma variabilidade muito menor do que a observada nos outros grupos. É importante ressaltar que o grupo TA apresentou sete terapeutas, dois dos quais são terapeutas de cinco salas (de um total de 10 analisadas). Dessa forma, esses dados tornam-se mais enviesados por características individuais desses dois participantes do que nos outros grupos analisados.

\section{DISCUSSÃO}

\section{Participantes}

O número de participantes inscritos neste estudo foi muito superior ao inicialmente estimado. Mesmo após o encerramento das inscrições, muitos indivíduos continuaram a tentar inscrever-se, muito embora no site estivesse claro que essas haviam sido encerradas.

Existiram muitas desistências anteriores à terapia, possivelmente devido à exigência de novas respostas dos participantes. A taxa de abandono encontrada, entretanto, está dentro do que análises desse fenômeno têm encontrado. No artigo de Lhullier e Nunes (2004) a taxa de abandono indicada vai de 30 a $60 \%$.

Neste estudo, verificaram-se as afirmações de Miller e Gergen (1998) sobre o grande potencial de alcance geográfico da terapia via Internet, já que os participantes eram provenientes de quase todos os Estados do Brasil, além de quatro que eram de outros países.
Pesquisas futuras devem considerar a possibilidade de utilização da terapia via Internet por indivíduos que residem em outros países e não têm acesso à terapia em sua própria língua, além de verificar sua utilidade para os indivíduos que não querem ou não podem, por motivos práticos ou de saúde, realizar a psicoterapia tradicional, sendo que tais estudos devem coletar essas informações no período de inscrição da pesquisa.

\section{Relação Terapêutica na Terapia via Internet}

A partir da aplicação do WAI nos três momentos da terapia e da divisão dos participantes em quatro grupos, foi realizada uma série de testes e análises estatísticas, cujos resultados serão discutidos de acordo com as características psicométricas do WAI, com o momento de formação da relação terapêutica, com a manutenção dessa relação ao longo do tempo, com as diferenças entre os grupos de terapeutas e clientes na predição de resultados e, ainda, com o comportamento dos terapeutas, sob o ponto de vista dos clientes, relativamente à força da relação terapêutica.

\section{Características Psicométricas do WAI}

Foram encontradas correlações positivas entre as subescalas, as quais variaram entre moderada e forte em todos os grupos, tendo havido diferenças, em todos os grupos, em pelo menos uma subescala, quando comparada às outras. A consistência interna (alpha) entre as subescalas do instrumento foi alta nos três momentos de aplicação.

Esses dados estão em concordância com o que Horvath e Greenberg (1989) e Horvath (1994) descreveram a respeito da intercorrelação entre as subescalas e o alto grau de consistência e confiabilidade do instrumento. Além disso, a diferença entre as subescalas reforça o que é dito por esses mesmos autores sobre $\mathrm{o}$ fato de as subescalas abordarem dimensões distintas da relação terapêutica e serem, apesar disso, altamente relacionadas entre si.

\section{Momento da Formação e Manutenção da Relação Terapêutica}

A literatura descreve que a relação forma-se no início da terapia, por volta da terceira ou quarta sessão (Garfield, 1995; Horvath, 1994; Luborsky, 1994). Neste estudo, verificou-se que a partir da $5^{\text {a }}$ semana de terapia a relação terapêutica havia se formado, e manteve-se estável no decorrer das 15 semanas de terapia (observando-se os grupos como um todo). Optou-se por medi-la por volta da $5^{a}$ semana, pois se levou em conta que nas primeiras semanas os 
participantes, além de iniciarem o contato terapêutico, também estariam adaptando-se ao sistema de comunicação.

Verificou-se, também, que a intensidade da relação terapêutica, para os clientes que terminaram a terapia, foi similar aos resultados de Al-Darmaki e Kivlighan (1993), Connors e cols. (1997), Kivlighan e cols. (1991), Kokohovic e Tracey (1990), Mallinckrodt, Coble e cols. (1995), Mallinckrodt, Gantt e cols. (1995) e Multon e cols. (1996), quando observados como um todo. As médias dos resultados do WAI deste estudo variaram entre 5.40 e 5.60 , enquanto os valores encontrados na literatura variam de 5.16 a 6 . Os valores da subescala Vínculo variaram entre 5.79 e 5.88 e os valores encontrados na literatura variam entre 5.59 e 5.90. Os valores da subescala Tarefa variaram entre 5.41 e 5.54, ao passo que os valores da literatura variaram entre 5.53 e 5.66. Os valores da subescala Objetivos variaram entre 5.05 e 5.31 e os encontrados na literatura variam entre 5.43 a 5.52 .

Ainda que essa análise seja meramente visual, pode-se observar que os valores da subescala Vínculo são os mais altos e, também, são os que estão mais próximos do intervalo de resultados encontrados na literatura. Isso indica que é possível estabelecer um forte vínculo de confiança, colaboração e comprometimento entre cliente e terapeuta na terapia via Internet. Também é possível que o limite estabelecido de 15 semanas tenha afetado negativamente a concordância entre terapeuta e clientes sobre os objetivos e tarefas da terapia.

Horvath (1994) argumenta que as relações terapêuticas têm características essenciais semelhantes no início da terapia e que o ambiente terapêutico torna-se mais complexo e específico na medida em que a terapia move-se em direção a fases posteriores. No presente estudo não foram analisadas terapias individualmente, entretanto observam-se mudanças sensíveis, porém não significativas, nos resultados do WAI e das subescalas no decorrer das 15 semanas entre os grupos de participantes que finalizaram a terapia. Análises e estudos futuros são necessários para observar melhor a questão do desenvolvimento, ao longo do tempo, da relação terapêutica, considerando as terapias individualmente.

\section{Diferenças entre grupos e predição de resultados}

Foram encontradas diferenças significativas entre os clientes que abandonaram a terapia comparativamente aos outros três grupos analisados, sendo que os resultados do WAI desse grupo foram mais baixos do que oso observados nos outros grupos.
Dessa forma, verifica-se que o WAI foi preditor de participação na terapia relativamente aos resultados dos clientes. Isso está de acordo com o que foi dito por Garfield (1995), Horvath (1994), Horvath e Greenberg (1989), Horvath e Symonds (1991), e Luborsky (1994) sobre medir a relação no início da terapia como forma de prever resultados.

Foi verificado, de acordo com o relatado por Horvath e Symonds (1991) e Luborsky (1994), que a visão dos clientes é melhor preditor de resultados do que a visão dos terapeutas. No presente estudo não foi constatado que a visão dos terapeutas seja preditor de resultados, o que não está em desacordo com a literatura. É necessário levar em conta, também, que os resultados do grupo dos terapeutas que atenderam as salas onde ocorreram os abandonos ficaram enviesados pela visão de dois terapeutas que foram responsáveis por cinco das dez salas avaliadas. Um dos terapeutas foi quem abandonou suas três salas e o outro foi o que teve a taxa maior de abandono de clientes atendidos.

Dessa forma, observa-se que para a amostra de participantes que terminaram a terapia, a relação terapêutica formou-se e manteve-se ao longo da terapia com características muito semelhantes às descritas na literatura de psicoterapia baseada em evidências e a respeito do inventário.

\section{CONCLUSÕES}

Em relação aos objetivos e hipóteses deste estudo, sendo observadas as limitações relativas ao tamanho da amostra pesquisada, ao tempo de tratamento e ao tipo de instrumento utilizado, conclui-se, com cautela, que: a relação terapêutica formou-se e manteve-se com características semelhantes às descritas na literatura, mostrando que, via Internet e por meio de formas de comunicação assíncronas, é possível estabelecer um clima produtivo entre terapeutas e clientes. Sendo a relação terapêutica um aspecto central da psicoterapia, a possibilidade de essa estabelecer-se via Internet indica que é possível existir psicoterapia por tal via.

É necessário que outros estudos possam verificar as diferenças entre a terapia via Internet nas diversas formas de comunicação eletrônica existentes e para que tipo de problemas e situações ela mostra-se mais útil. Também é importante ressaltar que a avaliação da relação terapêutica foi baseada na utilização do WAI e é necessário que outras formas de avaliação sejam utilizadas em estudos futuros. Métodos qualitativos podem ser utilizados para avaliar melhor e de forma mais individualizada a riqueza do processo terapêutico. 


\section{REFERÊNCIAS}

Al-Darmaki, F. \& Kivlighan, D. M. (1993). Congruence in client-counselor expectations for relationship and the working alliance. Journal of Counseling Psychology, 40, 379-384.

Barak, A. \& Wander-Schwartz, M. (1999, Aug.). Empirical evaluation of brief group therapy through an internet chat room. In S. A. King (Chair), Internet support groups: Group therapy by e-mail and chat. Symposium at the annual convention of the American Psychological Association, Boston.

Connors, G. J., Carroll, K. M., DiClemente, C. C., Longabaugh, R. \& Donovan, D. M. (1997). The therapeutic alliance and it's relationship to alcoholism treatment participation and outcome. Journal of Consulting and Clinical Psychology, 65, 588-598.

Conselho Federal de Psicologia (2000). Especifica e qualifica a psicoterapia como prática do psicólogo. Resolução CFP N.o 010/00 de 20 de dezembro de 2000.

Cook, J. (2001). Working alliance and internet therapy: comparison across modalities. Master Dissertation. Lewis \& Clark College, Portland.

Day, S. X. \& Schneider, P. L. (2002). Psychotherapy using distance technology: A comparison of face-to-face, video and audio treatment. Journal of Counseling Psychology, 49, 499-503.

Garfield, S. L. (1995, $2^{\mathrm{a}}$ ed.) The Therapy Client-Patient. In S. L. Garfield (Eds.), Psychotherapy: An eclectic and integrative approach. (pp. 39-65). New York: Wiley \& Sons.

Horvath, A. O. (1994). Empirical validation of Bordin's pantheorical model of the alliance: The working alliance inventory perspective. In A. O. Horvath, \& L. Greenberg (Eds.), The working alliance: Theory, research, and practice. (pp. 85 - 108). Nova York: John Wiley \& Sons.

Horvath, A. O. \& Greenberg, L. (1989). Development and validation of the Working Alliance Inventory. Journal of Counseling Psychology, 41, 438-448.

Horvath, A. O. \& Symonds, B. D. (1991). The relation between working alliance and outcome in psychotherapy: A meta-analysis. Journal of Counseling Psychology, 38, 139-149.

Keller, F. S (1983). Psicologia: problemas históricos. Em R. R. Kerbauy (Org.), Fred Simmons Keller. (pp. 99- 127). São Paulo: Ática.
King, S. A. (1998). Internet therapy and self help groups: The pros and cons. In J. Gackenbah (Ed.), Psychology and the internet: Intrapersonal, interpersonal, and transpersonal implications. (pp. 77-110). San Diego: Academic.

Kivlighan, D. M., Angelone, E. O. \& Swafford, K. G. (1991). Live supervision in individual psychotherapy: Effects on therapist's intention use and client's evaluation of session effect and working alliance. Professional Psychology: Research and Practice, 22(6), 489-495.

Kokohovic, A. M. \& Tracey, T. J. (1990). Working alliance in the early phase of counseling. Journal of Counseling and Psychology, 37(1), 16-22.

Lhullier, A. C. \& Nunes, M. L. T. (2004). Uma aliança que se rompe. Ciência e Profissão: Diálogos, 1, 43-49.

Luborsky, L. (1994). Therapeutic alliances as predictors of psychotherapy outcomes: Factors explaining the predictive success. In A. O. Horvath \& L. Greenberg (Eds.), The working alliance: Theory, research, and practice. (pp. 39 - 50). Nova York: John Wiley \& Sons.

Mallinckrodt, B., Coble, H. M. \& Gantt, D. L. (1995). Working alliance, attachment memories, and social competencies of women in brief therapy. Journal of Counseling Psychology, 42, 79-84.

Mallinckrodt, B., Gantt, D. L. \& Coble, H. L. (1995). Attachment patterns in the psychotherapy relationship: Development of the client attachment to therapist scale. Journal of Counseling Psychology, 42, 307-317.

Meyer, S. B. \& Vermes, J. S. (2001). Relação terapêutica. Em B. Rangé (Org.), Psicoterapias CognitivoComportamentais: um diálogo com a psiquiatria. (pp. 101-110). Porto Alegre: Artmed.

Miller, J. K. \& Gergen, K. J. (1998). Life on the line: The therapeutic potentials of computer-mediated conversation. Journal-of-Marital-and-Family-Therapy, 24(2), 189-202.

Multon, K. D., Kivlighan, Jr., D. M. \& Gold, P. (1996). Changes in counselor adherence over the course of training. Journal of Counseling Psychology, 43, 356-363.

Sander, F. M. (1996). Couples group therapy conducted via computer-mediated communication: A preliminary case study. Computers-in-Human-Behavior, 12(2), 301-312.

Recebido em 26/04/2004 Aceito em 01/03/2006

Endereço para correspondência: Oliver Zancul Prado. Rua Rui Barbosa 1277, CEP 13560-330, São Carlos-SP.

E-mail: ozp@psico.net 